\title{
Effectiveness Factor Calculations for Immobilized Enzyme Catalysts
}

DAVID J. FINK, Department of Chemical Engineering, TSUNGYEN NA, Department of Mechanical Engineering, University of Michigan-Dearborn, and JEROME S. SCHULTZ, Department of Chemical Engineering, University of Michigan, Ann Arbor, Michigan 48104

\section{Summary}

The steady state, nonlinear diffusion equations which describe reactions in constrained enzyme solutions are of great interest in many biological and engineering applications. As in other types of nonlinear differential equations, exact analytical solutions do not exist except in some simplified cases. In this paper, a general procedure is presented for solving numerically for the substrate concentration profile and effectiveness factor utilizing the transformation method suggested by $\mathrm{Na}$ and $\mathrm{Na}$. Design correlations for enzyme solutions constrained within spherical membranes are included. The use of a unique definition of the Thiele Modulus in these charts permits the clear illustration of the effects of substrate concentration and external mass transfer resistances on the overall effectiveness factor for the catalyst particle.

\section{INTRODUCTION}

The steady state, nonlinear diffusion equations which describe reactions in constrained enzyme solutions are of great interest in many biological and engineering applications. As in other types of nonlinear differential equations, exact analytical solutions do not exist except in some simplified cases.

Rony ${ }^{1}$ recently provided relationships for calculating effectiveness factors which accounted for both internal and external diffusion resistances. These equations, for planar, cylindrical, and spherical membrane systems, are restricted to first-order kinetics. Kobayashi and Moo-Young ${ }^{2}$ have given equations for maxtrix-bound enzymes, but only external transfer resistances are considered. In another study, ${ }^{3}$ they provided solutions for several enzyme kinetic expressions, but only for planar membranes with internal transfer resistances. 
Thus the general problem of simultaneous internal and external mass transfer resistances with Michaelis-Menten kineties, and either planar, cylindrical, or spherical geometries has not been previously treated.

In this paper, a general procedure is presented for solving numerically for the substrate concentration profile and effectiveness factor utilizing the transformation method suggested by $\mathrm{Na}$ and $\mathrm{Na} .{ }^{4}$ Effectiveness correlations for enzyme solutions constrained within spherical membranes are given to illustrate the method. The use of a unique definition of the Thicle Modulus in these charts permits the clear illustration of the offects of substrate concentration and external mass transfer resistances on the overall effectiveness factor for the catalyst particle.

\section{PROBLEM DESCRIPTION}

The idealized system to be considered here is that of a homogeneous enzyme solution entrapped within a permeable membrane as shown in Figure 1(a). The membrane is permeable to small substrate molecules but impermeable to larger enzymes, and is surrounded by an infinite substrate solution. All reaction is confined to the region bounded by the mombrane $\left(r<r_{0}\right)$. The reaction mechanism is shown in Figure 1(b). External substrate molecules $\left(S_{e}\right)$ diffuse through the external liquid film $\left(R_{0}<r<R_{\infty}\right)$ and the membrane $\left(r_{0}<r<R_{0}\right)$. The internal substrate $\left(S_{i}\right)$ couples with the enzyme and reacts to form product $\left(P_{i}\right)$. The product then diffuses out of the membrane-bounded region.

A typical substrate concentration profile is shown in Figure 1(c). Changes in $c_{s}$, which occur at $r_{0}$ and $R_{0}$, indicate the possibility of partitioning of the substrate at the membrane-liquid interfaces. Note that by setting $r_{0}=R_{0}$, the model system described above can also be used to represent matrix-bound enzyme systems in which the enzyme is homogencously distributed in the matrix phase and an "apparent diffusivity" is defined for the substrate in the catalyst particle.

The following assumptions will be made in the problem development: 1) The enzyme is homogeneously distributed throughout the interior of the coll. 2) The system is at steady state. 3) Enzyme reaction kinetics can be represented by an expression of the Michaelis-Menten type with respect to the substrate concentration, i.e., the volumetric rate of disappearance of $S_{i}$ is

$$
v_{\mathrm{s}}=k_{2} E_{\iota} c_{\mathrm{is}} /\left(K+c_{\mathrm{is}}\right)
$$




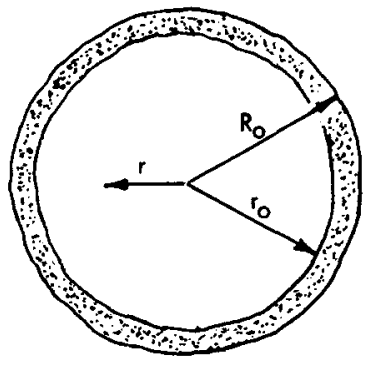

(a)

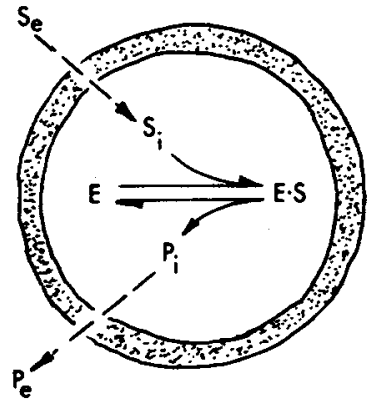

(b)

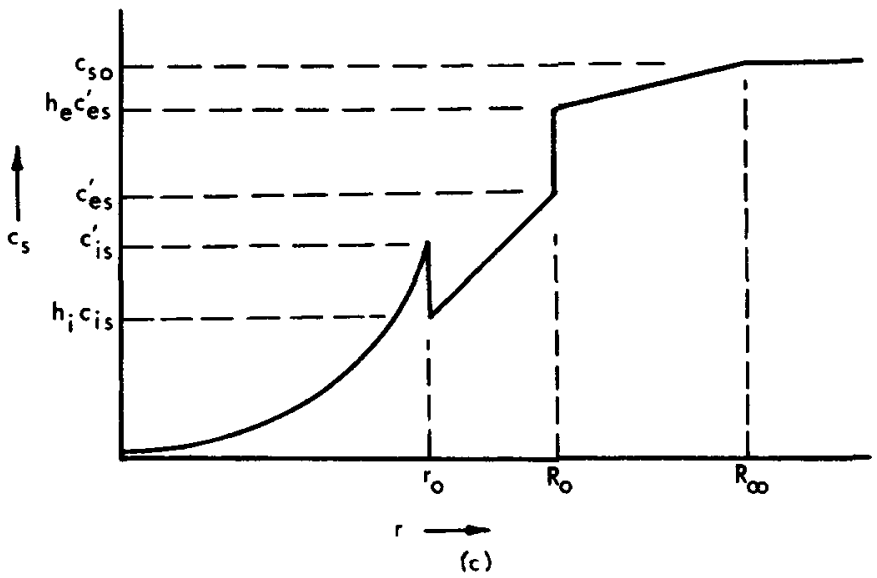

Fig. 1. Description of immobilized enzyme system: (a) cell geometry; (b) reaction mechanism; (c) substrate concentration profile.

Several mechanisms exist $\mathrm{t}^{3,5}$ which produce kinetic expressions of the type given in eq. (1). 4) The internal substrate diffusivity, $D_{\text {is }}$, is the same for all $r<r_{0}$. 5) The presence of reaction products does not affect the rate of substrate reaction.

A differential mass balance for the substrate leads to the following governing equation in the region where the enzyme is distributed $\left(r<r_{0}\right)$ :

$$
D_{\mathrm{is}}\left[\frac{d^{2} c_{\mathrm{is}}}{d r^{2}}+\frac{(p+1)}{r} \frac{d c_{\mathrm{is}}}{d r}\right]=\frac{k_{2} E_{t} c_{\mathrm{is}}}{K+c_{\mathrm{is}}}
$$


In eq. (2), $p$ is a geometric factor indicating the type of geometry which applies; $p=-1,0$, and +1 for planar, cylindrical, and spherical membranes, respectively. The distance dimension, $r$, represents the radial coordinate for $p=0$ or +1 , and the coordinate perpendicular to the membrane for $p=-1$.

The boundary conditions which apply are:

$$
\begin{aligned}
r=r_{0}: N_{s} & =D_{\mathrm{is}}\left(d c_{\mathrm{is}} / d r\right) \\
& =P_{s}\left(c_{\mathrm{es}}^{\prime}-h_{i} c_{\mathrm{is}}^{\prime}\right) \\
& =k_{e}\left(A_{R_{0}} / A_{r_{0}}\right)\left(c_{\mathrm{s} 0}-h_{e} c^{\prime}{ }_{\mathrm{es}}\right) \\
& =P\left[\left(c_{\mathrm{s} 0} / h_{e}\right)-h_{i} c_{\mathrm{is}}\right]
\end{aligned}
$$

where

$$
\begin{gathered}
1 / P=\left(A_{r_{0}} / A_{R_{0}}\right)\left(1 / h_{e} k_{e}\right)+1 / P_{s} \\
r=0: d c_{\mathrm{is}} / d r=0 \quad \text { and } \quad 0 \leq c_{\mathrm{is}} \leq c_{\mathrm{g} 0}
\end{gathered}
$$

$N_{s}$ is the flux of substrate into the enzyme region, evaluated at $r=r_{0}$. The factor $A_{r_{0}} / A_{R_{0}}$ is the ratio of the membrane area at $r=r_{0}$ to the area at $r=R_{0}$. Values for $k_{e}$, the external mass transfer coefficient, can be estimated from correlations such as those presented by Satterfield. ${ }^{5}$

The apparent externally observed, volumetric rate of reaction within the confined enzyme phase, $v_{e s}$, is given by eq. (3).

$$
v_{\text {es }}=\left(A_{r_{0}} / V_{r_{0}}\right) \cdot N_{s}=\left[(p+2) / r_{0}\right] N_{s}
$$

The following quantities will be used to generalize the analysis to follow:

$$
\begin{aligned}
\kappa & =K / c_{\mathrm{s} 0} \\
\beta & =k_{2} E_{\imath} r_{0}^{2} / D_{\mathrm{is}} K \\
\alpha & =P r_{0} / D_{\mathrm{is}} \\
\eta_{0} & =v_{\mathrm{es}} /\left[k_{2} E_{t} c_{\mathrm{s} 0} /\left(K+c_{\mathrm{s} 0}\right)\right] \\
\phi & =\left[r_{0}{ }^{2} k_{2} E_{t} / D_{\mathrm{is}}\left(K+c_{\mathrm{s} 0}\right)\right]^{1 / 2} \\
& =\left[\beta_{\kappa} /(1+\kappa)\right]^{1 / 2}
\end{aligned}
$$

$\beta$ is a Damköhler number for the enzyme phase with $K$ used as the characteristic concentration. The Sherwood number, $\alpha$, is defined for combined membrane and external film mass transfer. $\eta_{0}$, the "overall effectiveness factor" as defined by Satterfield, ${ }^{5}$ is the ratio 
of the actual reaction rate to the uniform rate that would be observed at the external substrate concentration, i.e., in the absence of diffusional resistances. The new Thiele Modulus, as we have defined it in eq. (4e), conveniently includes the transition from first- to zero-order kinetics as the external concentration, $c_{\mathrm{B} 0}$, is increased. The present authors tend to agree with Satterfield (ref. 5, p. 202) that the use of more complicated "asymptotic" moduli ${ }^{3,6}$ results in a loss of simplicity and physical meaning in defining the system kinetics.

Equation (2) can now be cast in dimensionless form as

$$
\left(d^{2} c / d x^{2}\right)+[(p+1) / x](d c / d x)=\beta \kappa[c /(c+\kappa)]
$$

where the dimensionless variables are $x=r / r_{0}$ and $c=c_{\mathrm{is}} / c_{\mathrm{s} 0}$. The boundary conditions become

$$
\begin{aligned}
& x=1:(d c / d x)=\alpha\left[\left(1 / h_{e}\right)-h_{i} c(1)\right] \\
& x=0:(d c / d x)=0 \text { and } \quad 0 \leq c \leq 1
\end{aligned}
$$

$\eta_{0}$ is found by combining eqs. (2a), (3), and (4d) to give

$$
\eta_{0}=\left.\left\{[(p+2)(1+\kappa)] / \beta_{\kappa}\right\}(d c / d x)\right|_{x=1}
$$

\section{NUMERICAL COMPUTATION PROCEDURE}

The transformation proposed by $\mathrm{Na}$ and $\mathrm{Na}^{4}$ permits the rewriting of the two-point, boundary value problem of eq. (6) into an initial value problem. The transformation amounts to stretching the concentration coordinate by defining

$$
c=A c^{*} \quad \text { or } \quad c^{*}=c / A
$$

where $A$ is the transformation parameter. When $c^{*}$ at $x=0$ is set equal to 1 , resulting in $A=c(x=0)$, eq. (5) is transformed to

$$
d^{2} c^{*} / d x^{2}+[(p+1) / x]\left(d c^{*} / d x\right)=\beta_{\kappa}^{*}\left[c^{*} /\left(c^{*}+\kappa^{*}\right)\right]
$$

where $\kappa^{*}=\kappa / A$. The transformed boundary conditions are

$$
\begin{aligned}
& x=1:\left(d c^{*} / d x\right)=\alpha\left[\left(1 / A h_{e}\right)-h_{i} c^{*}(1)\right] \\
& x=0:\left(d c^{*} / d x\right)=0 \quad \text { and } \quad c^{*}=1
\end{aligned}
$$

The numerical integration of eq. (5) then proceeds in the following manner:

1) Values for $\beta$ and $\kappa^{*}$ are selected.

2) Equation (8) is integrated as an initial value problem from $x=0$ to $x=1$ to determine $c^{*}$ and $\left(d c^{*} / d x\right)$ at $x=1$. Intermediate values of $c^{*}$ are saved as desired. 
3) A range of values of $\alpha$ can be chosen and then the corresponding values of $A$ are calculated from eq. (8a) as

$$
A=1 /\left[h_{i} c^{*}(1)+\left.(1 / \alpha)\left(d c^{*} / d x\right)\right|_{x=1}\right] h_{e}
$$

4) The variables can then be retransformed by eq. (7) to the unstretched coordinates to determine the actual concentration profiles and the values of $k$. In this way, $\eta_{0}$ can be calculated from eq. (6) for a given $\beta$, and a range of values of $\alpha$ and $\kappa$.

An alternate calculation algorithm would be the following:

1) Values for $c(0), \beta$, and $\alpha$ are selected.

2) Equation (5) is integrated from $x=0$ to $x=1$ to find $c$ and $(d c / d x)$ at $x=1$.

3) The value of $\alpha$ corresponding to the assumed set of parameter values can then be calculated from eq. (5a):

$$
\alpha=\left.(d c / d x)\right|_{x=1} /\left[\left(1 / h_{e}\right)-h_{i} c(1)\right]
$$

This procedure then gives $\eta_{0}$ versus $\alpha$ for fixed values of $\beta$ and $\kappa$. The later algorithm requires many more computations than the former one to produce the same information, since eq. (5) must be integrated for each set of parameter values.

Application of l'Hospital's rule to eq. (8) leads to the following relationship for $\left(d^{2} c^{*} / d x^{2}\right)$ at $x=0$, needed to start the numerical integration:

$$
\left.\left(d^{2} c^{*} / d x^{2}\right)\right|_{x=0}=\left[\beta \kappa^{*} /(p+2)\right]\left[c^{*} /\left(c^{*}+\kappa^{*}\right)\right]
$$

Thus the numerical integration difficulties encountered at $x=0$ by Moo-Young and Kobayashi ${ }^{3}$ are not found if the transformation procedure discussed above is employed.

Integration of eq. (5) subject to boundary conditions (5a) and (5b) has been carried out for many values of $\beta$ and $\alpha$. A double precision, four-point Runge-Kutta integration routine with grid spacing of $\Delta x=0.01$ was used in all cases.

\section{GENERALIZED EFFECTIVENESS FACTOR CHARTS}

The generalized effectiveness factor charts presented in Figures 2 and 3 are intended to permit rapid estimation of effectiveness factors in spherical, immobilized enzyme particles when $h_{i}$ and $h_{e}$ are unity. This information should enable the reactor designer to quickly decide which parameters are important in a specific application and possibly simplify the design analysis. 


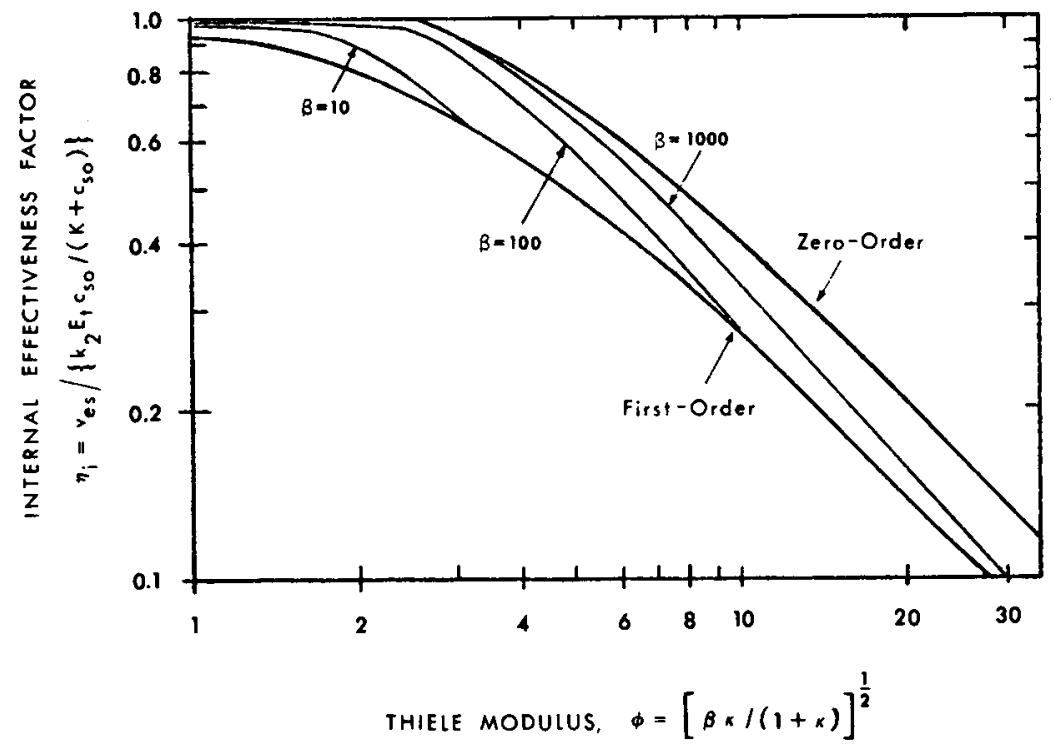

Fig. 2. Effectiveness factor for Michaelis-Menten enzymes encapsulated in spheres for $h_{i}=h_{e}=1$. $\beta=\left(k_{2} E_{t} r_{0}^{2}\right) /\left(K_{m} D_{\mathrm{is}}\right)$ and $\kappa=K_{m} / c_{\mathrm{s} 0}$. The effect of the external film and membrane resistances has been neglected ( $\alpha=P r_{0}$ / $D_{\mathrm{is}}=\infty$ ).

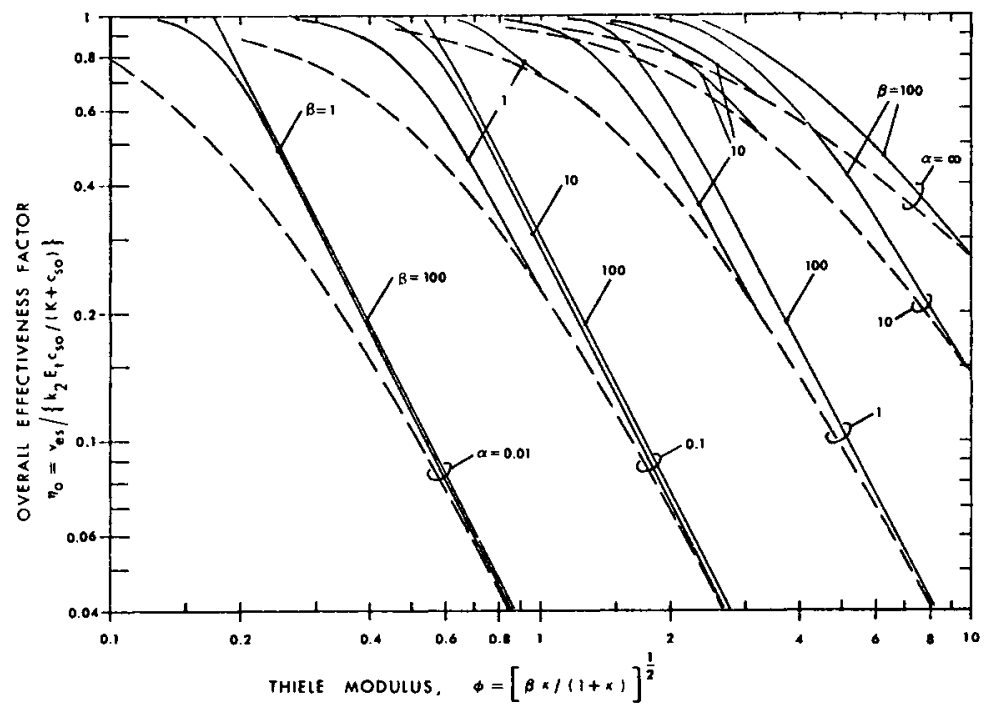

Fig. 3. Overall effectiveness factor for Michaelis-Menten (solid lines) and first-order (dashed lines) reactions in spheres for $h_{i}=h_{e}=1$. $\beta=k_{2} E_{t} r_{0}{ }^{2} /$ $K D_{\mathrm{is}, \kappa}=K / c_{\mathrm{B} 0}$, and $\alpha=P r_{0} / D_{\mathrm{ig}}$. 
The chart presented in Figure 2 gives the effectiveness factor, $\eta_{1}$, for the internal, enzyme-containing phase only. The upper and lower bounds for $\eta_{\mathrm{I}}$ are provided by assuming zero- and first-order kinetics in eq. (5), respectively.

The definition of $\phi$ as given in eq. (4e) permits one to follow the effect of external substrate concentration in Figure 2 as lines of constant $\beta$ since a given encapsulated enzyme will have a characteristic value of $\beta$. If $\beta$ is held constant, then a change in the external substrate concentration will be reflected in a change in the Thiele Modulus since $\kappa=K / c_{\mathrm{s} 0}$. In Figure 2 , for example, with $\beta=100$ and low substrate concentrations (large $K$, first-order kinetics), the effectiveness factor approaches a limit of 0.27 at $\phi=\beta^{1 / 2}=10$. For high $c_{\mathrm{s} 0}$ (low $K$, zero-order kinetics), $\eta_{\mathrm{I}}$ is greater than 0.98 for $\phi<2$. For this particular enzyme system, $\beta=100$, the internal effectiveness factor varies with external concentration by a range of over threefold between the limits of first and zero-order kinetics.

Overall effectiveness factors, $\eta_{0}$, which include internal and external diffusion resistances calculated by the algorithm presented above are given in Figure 3.* The parameter $\alpha$ gives the relative magnitude of internal to external diffusion resistances. The presence of mass transfer resistances surrounding the enzyme zone can greatly affect the efficiency of the catalyst. For example, considering the same enzyme particle as above $(\beta=100)$ and $\alpha=1$, the overall effectiveness factor for spheres can vary from 0.98 to 0.027 , or over a range of thirtyfold, as the external substrate concentration is changed. The need for the exact numerical solutions for estimating the performance of immobilized enzyme catalysts is evident, therefore, when $\beta$, as defined here, becomes greater than about 10 for spherical particles. When the overall effectiveness factor drops below about 0.1 , however, the curves in Figure 3 suggest that little error will be incurred by using first-order kinetics to approximate Michaelis-Menten kinetics.

\section{CONCLUSIONS}

An efficient algorithm has been presented for numerically solving the diffusion-reaction equations for Michaelis-Menten enzymes. Solution of these equations has led to generalized charts which give the overall effectiveness factor for spherical catalyst particles as a function of system parameters. A unique definition of the Thiele Modulus permits the clear illustration of the effects of bulk substrate

* Similar charts to Figures 2 and 3 for planar and cylindrical coordinates are available from the authors. 
concentration and external mass transfer limitations on the effectiveness factor. These charts are useful in quickly determining the important design parameters for immobilized enzyme reactors. They can also easily be used to check the approximate validity of kinetic constants calculated from experimental data.

\section{Nomenclature}

A transformation parameter

$A_{\text {r } 0} \quad$ area of capsule membrane at $r_{0}, \mathrm{~cm}^{2}$

$A_{R 0} \quad$ area of capsule membrane at $R_{0}, \mathrm{~cm}^{2}$

$c \quad$ dimensionless substrate concentration

$c^{*} \quad$ transformed dimensionless substrate concentration

$c^{\prime}{ }_{e s} \quad$ substrate concentration at outer surface of cell membrane, $\mathrm{m} M$

$c^{\prime}$ is substrate concentration at inner surface of cell membrane, $\mathrm{m} M$

$c_{\text {is }} \quad$ substrate concentration inside cell, $\mathrm{m} M$

$c_{80} \quad$ bulk substrate concentration at $r=R_{\infty}, \mathrm{m} M$

$D_{\text {is }} \quad$ internal substrate diffusivity, $\mathrm{cm}^{2} / \mathrm{sec}$

$E_{t} \quad$ total enzyme concentration, $\mu M$

$h_{e}, h_{i} \quad$ external and internal partition coefficients for membranes

$k_{e} \quad$ external mass transfer coefficient, $\mathrm{cm} / \mathrm{sec}$

$k_{i}, k_{-1}, k_{-2}$ reaction rate constants in Michaelis-Menten mechanism

$K \quad$ Michaelis-Menten constant, $\mathrm{m} M$

$N_{s} \quad$ flux of substrate across membrane into the cell, defined at $r=r_{0}$, $\mu \mathrm{moles} / \mathrm{cm}^{2} / \mathrm{sec}$

$p \quad$ geometric factor indicating planar, cylindrical, or spherical coordinates

$P \quad$ total substrate permeability coefficient for membrane and external film evaluated based on $A_{r 0}, \mathrm{~cm} / \mathrm{sec}$

$P_{8} \quad$ substrate permeability coefficient for membrane, $\mathrm{cm} / \mathrm{sec}$

$r \quad$ distance coordinate, $\mathrm{cm}$

$r_{0} \quad$ radius or depth of enzyme-containing region, $\mathrm{cm}$

$R_{0} \quad$ outer radius of membrane, $\mathrm{cm}$

$R_{\infty} \quad$ outer radius of external film, $\mathrm{cm}$

$v_{\text {es }} \quad$ externally observed volumetric rate of consumption of substrate, $\mu$ moles $/ \mathrm{cm}^{3}$ of catalyst/sec

$v_{a} \quad$ local volumetric rate of consumption of substrate, $\mu$ moles $/ \mathrm{cm}^{3} / \mathrm{sec}$

$V_{r 0} \quad$ volume of enzyme phase enclosed by membrane, $\mathrm{cm}^{3}$

$x \quad$ dimensionless distance coordinate

$\alpha \quad$ Sherwood number for membrane transport

$\beta \quad$ Damköhler number for enzyme phase

$\eta_{0} \quad$ overall effectiveness factor for catalyst particle

$\eta_{\mathrm{I}} \quad$ effectiveness factor for catalyst in absence of membrane or external film mass transfer resistances

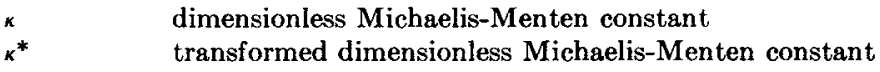

$\phi \quad$ Thiele Modulus in eq. (4e)

This work was supported in part by Public Health Service Grant GM 15152 and a Research Career Development Award No. 1K04GM08271 from the Insti- 
tute of General Medical Sciences to J.S.S. The support of the Dutch Science Foundation, Z.W.O., while J.S.S. was a visiting professor at the University of Nijmegen is gratefully acknowledged.

\section{References}

1. P. R. Rony, Biotechnol. Bioeng., 13, 431 (1971).

2. T. Kobayashi and M. Moo-Young, Biotechnol. Bioeng., 13, 893 (1971).

3. M. Moo-Young and T. Kobayashi, Can. J. Chem. Eng., 50, 162 (1972).

4. H. S. Na and T. Y. Na, Math. Biosci., 6, 25 (1970).

5. C. N. Satterfield, Mass Transfer in Heterogeneous Catalysis, M.I.T. Press, Cambridge, Mass., 1970.

6. K. B. Bischoff, AIChE J., 11(2), 351 (1965).

Accepted for Publication March 31, 1973 